\title{
Effectively Cultivating Undergraduate Ability of Information Management and Information System Specialty in the Context of Big Data
}

\author{
Hejie Chen ${ }^{1,2}$, Lidaishan $\mathrm{Yu}^{2}$ \\ 1School of Economics and Management, Beijing University of Post and Telecommunications, Beijing \\ chenhejie81@sina.com \\ 2 School of Economics and Management, Beijing Institute of Graphic Communication, Beijing \\ yulidaishan@163.com
}

\begin{abstract}
Data science has become an important direction of management science. The wide application of big data analysis in business brought new challenges to the information management and information system specialty. According to training program of our college, this paper describes a curricular framework of computer science and data science. It considers the professional skills training of information management and information specialty, and also considers the cultivation ability of programming language of big data and data analysis. The courses divide into two threads in accordance with computer thread and big data thread. The former focuses on the computer theory and operation abilities. The latter emphasizes programming and data analysis science of data science. The paper also introduced several effective pedagogical methods that combine with big data. Experimentation is an important ability cultivating mean, which train students a high level of application ability. So this paper introduces experimentation arrangement.
\end{abstract}

Keywords-Big data; Computer courses curriculum; Information management and information system specialty; Curriculum introduction; Experimentation arrange

\section{INTRODUCTION}

Information management and Information system specialty is one specialty of management department of Beijing Institute of Graphic Communication [1]. In the undergraduate education system in the United States, the Information management and Information system specialty (abbreviation is IMISS) is generally a part of the information science, with a focus on IT technology. However IMISS is a part of the management science, with a focus on management .After graduation, students are mainly engaged in the maintenance and management of enterprise information systems, and can make information recommendations. Therefore, the curriculum reflects the combination of management ideas and information systems, and students' programming skills are not high. With the rapid development of big data technology, the enterprise's core business (such as marketing) relied on data analysis to make decisions. Computer science, statistics science and management science of many universities set up big data in the United States [2] [3]. From the perspective of management science, the paper discussed the big data curriculum of the Beijing Institute of Graphic Communication. Our IMISS focused on the cross-knowledge of statistics science, computer science and management science. We adopted business and marketing examples into data science.
Information management and Information system specialty of the Beijing Institute of Graphic Communication is currently a total of 4 classes and 121 students. For educational reform, the first semester mainly introduced English, mathematics and other basic management courses. At the second semester, students learned the basic courses and management basic courses of Information management and Information system specialty. The third semester, they study professional courses of Information management and Information system specialty. And the fourth semester, students work in the enterprises.

Section 2 presents training objectives of curricular framework of management department in the context of big data. We also introduce teaching objective and content of some important courses in order to clarify our teaching philosophy. Section 3 introduces some effective pedagogical tools through teaching practices. Big data courses emphasize hands-on capacity, while the hands-on abilities rely mainly on curricular experimentation and the comprehensive experimentation. Therefore, Section 4 presents the experimental arrangement. At last, some conclusions and future works is introduced.

\section{CURRICULAR FRAMEWORK OF INFORMATION MANAGEMENT AND INFORMATION SYSTEM SPECIALTY IN THE CONTEXT OF BIG DATA}

The big data curriculum system belonged to management science included data acquisition, data cleansing, data analysis, and data visualization. Therefore, the students learned basic computer knowledge. For example, they gained the basic programming skills through $\mathrm{C}$. In the third semester, students mastered the knowledge of structured data management through database courses, which included the theoretical knowledge of relational databases and software operation capabilities. In the same semester, a data science course is offered to enable students to master the basic theoretical knowledge of data science. In the fourth semester, the Python language course is important programming languages of big data application. The students must expertly used Python language. The two courses of data analysis processing and data visualization processing ensure that students have various problems to solve big data applications of the enterprise.

The course divided into two threads: Computer thread and big data thread. Computer thread included basic computer courses (e.g. computer culture), electric commerce and enterprise management. Electric commerce focused on web technology (for example web programming, website 
design and implement). And enterprise management focused on the information system in enterprise application (for example MIS, ERP). We mainly describe some courses Computer Culture. big data thread focused on data analysis life cycle. In fact, some courses are structured data theories and software operations (for example database system).Other courses are semi-structured data. The latter emphasizes programming and data analysis capabilities without emphasizing hardware. The curricular framework is described in the figure1.The paper firstly introduced computer thread.

\begin{tabular}{|c|c|}
\hline \multicolumn{2}{|c|}{ Curricular Framework of IMISS } \\
\hline $\begin{array}{l}\quad \text { Computer Thread } \\
\text { Computer culture } \\
\text { Programming Language } \\
\text { Computer Network } \\
\text { Management Information } \\
\text { System } \\
\text { Electric commerce } \\
\text { Website programming }\end{array}$ & \begin{tabular}{|l}
\multicolumn{1}{|c}{ Big Data Thread } \\
Database System \\
Python \\
Data Science \\
Big Data Analysis \\
Web Scraping \\
Data Visualization \\
Data mining \\
Decision Support System
\end{tabular} \\
\hline
\end{tabular}

Fig. 1 Curricular Framework of IMMISS

\section{A. Computer Culture}

The computer culture is a primer basic course of the computer academics. This course involves the foundation knowledge; operate system, word processing, the electronics form and Internet technique of the computer. The undergraduate students learn this course; they systematically learn the basic computer knowledge and the operations of the computer. These technologies help the undergraduate students' self-study the computer courses in the future.

\section{B. Computer Network}

The goals of Computer Network are to train the networking users and the network-based application developers. In particular, our goals are: To understand how the global network infrastructure works. To understand how the network works well in today's world. To understand how the network works well in the future in the face of rapidly growing scale and heterogeneity. To understand the core ideas of Internet application programming be written.

\section{Programming Language}

Visual Basic Programming is selected as programming language. Visual Basic is the first visual development tool from Microsoft, and it was to compete with $\mathrm{C}, \mathrm{C}++$, Pascal and other well-known programming languages. It is the first programming language for our student Why do we choice Visual Basic? Visual Basic not only is a programming language, but also is a complete graphical development environment. This environment allows users with little programming experience to quickly develop useful Microsoft Windows applications which have the ability to use OLE (Object Linking and Embedding) objects. Visual Basic also has the ability to develop programs that can be used as a front end application to a database system, serving as the user interface which collects user.

\section{Electronic Commerce}

This is a lecture-based course that is designed to provide the student with thorough knowledge of e-commerce concepts and terminology. Topics include e-commerce applications, methodologies, and services address business solutions needed for electronic procurement, supplier management, and customer relationship management.

\section{E. Management Information System}

Management information system managed enterprise business, organization running used information technology. It provided a serious methods and tools to analyze business process, data process, system design, and system realization.

The following courses are the core courses of big data thread.

\section{F. Database Systems}

Every Company or government agency produces vast data every day. How to manage, save and use data? So, we choose Database Systems. This course introduces undergraduate students the foundations of database systems. The education goals of Database Systems are train some Database administrator and some user. The course focuses on the relational database system. Topics covered including: data models, relational data model; schema normalization and integrity constraints; query processing; recovery; isolation and consistency; security.

\section{G. Python}

The course included Python basics knowledge, data processing and data analysis using Python. The course first introduced the installation of Python; it then introduced the basic concepts and operations of Python, including lists etc.; introduced some advanced deepen concepts and operations, including abstractions, exceptions etc; finally introduced basics operating of data processing and data analysis.

\section{H. Data Science}

The main content included the knowledge of a big data analysis, various stages of the data analysis life cycle. Topics include: data science and workflow, statistical models and machine learning algorithms, information extraction and statistical variable creation, data visualization and social networking, predictive and causal analysis, data preprocessing and engineering methods.

\section{Big Data Analysis}

The course introduced the theories, methods, and tools of data mining, statistical learning, and pattern recognition of big data analytics. Then it introduced the use of Python to complete data processing, data analysis and so on. Also explain the use of Python on the operation of the database.

\section{J. Web Scraping}

This course is follow up coursed of python. The course explains techniques and methods for crawling web data and extracting information using the Python language. And the students mastered the basic capabilities of data crawling and web page parsing. The main content introduced data collection, data storage, dynamic website crawling, App crawling, verification code cracking, simulated login, proxy usage, crawler framework, distributed crawling, etc. 


\section{K. Data Visualization}

The course introduced a variety of graphical methods (such as column, pie, line, and scatter plots) and introduced commonly visualization software (such as $\mathrm{R}$, Illustrator). The course focused on data visualization using Python. The course introduced commonly used visual Python libraries such as Numpy, Scipy etc.

\section{Data Minding}

This course introduced the theories and software operation of data mining. It included four topics: data, classification, correlation analysis and clustering, and anomaly detection.

\section{Decision Support System}

Decision support system assisted the decision-makers to make decisions through the data, models and knowledge. DSS is a human-computer interaction for semi-structured and unstructured decision-making computer applications. DSS provided analyzing issue, constituting model, simulating decision. It used the various information resources and analysis tools to improve decision level and decision quality.

\section{Some Pedagogical Methods}

In order to increase practice ability, self-study ability and innovation ability of students, we have adopted a variety of pedagogical methods (for example a problem-based approach). These pedagogical methods effectively encouraged the students to independently think. And these methods mobilize initiative and enthusiasm of students to learn knowledge.

\section{A. A Problem-based Approach}

Problem-based learning attracted students' attention and induced learn interest. And it is an instructional method that is said to provide students with knowledge suitable for problem solving [1]. In practice, we use the problem-based lecture approach and the problem-based learning approach.

The former, the teacher asks a question and translates the knowledge surrounding the question. Sometimes, it used in conjunction with brainstorming. For example, when teaching big data definition, the teacher asked "What is the definition of big data? What is the difference between big data and relational databases?" Students provided many answers through brainstorming. In practice, we found that this method is more efficient than direct teaching by teachers.

The later, the students propose a solution according the question. This method is usually used to explain data analysis. The teacher proposed core questions of data analysis in order.The students answered questions, the solution of key problems of data analysis is also proposed. This approach broke a complex problem into many small problems. And students increased confidence when solving difficult problems.

\section{B. A Cooperative Learning Approach}

A cooperative learning is an instructional technique that requires students to work together in small, fixed groups on a structured learning task [2]. It can train positive interdependence and individual accountability. We usually adopt the cooperative learning approach to finish a project in comprehensive experimentation. For example, in the big data analysis, the teacher asked three students to form a group to use python to capture online data, data cleansing, data analysis, and writing a paper. Students are required to actively participate in each phase. The students assume different roles in each phase to allow them to experience different leadership responsibilities.

\section{A Case Study Approach}

A case study approach is widely adopted a pedagogical method by many coursed. When some theories are very complicated or difficult to understand, we use the case study approach. Case study is the study of the particularity and complexity of a single case, coming to understand its activity within important circumstances [3]. Selecting a case standard is that is easy to understand and comprehensively explanation this problem. The teacher looks for the detail of interaction with its context during teaching. For example, the teacher usually use marketing cases to explain the knowledge points of "The enterprise gained consumer purchase behavior through data analysis".

\section{EXPERIMENTATION ARRANGE}

IMISS emphasized different software operation ability. So, experimentation is an important actor in teaching. The key aim of experimentations is to ensure that students make an in-depth understanding theoretical content through operating software. Experimentation categories of IMISS are subdivided into three. They are the curricular experimentation, the comprehensive experimentation and emulation experimentation. The biggest problem we faced is limited experimental hours. We must arrange experimentation of traditional IMMS (for example he programming and management information systems), but also big data coursed experimentation.

The curricula experimentations were allocated within the 21-week semester. All three specialties launched the curricular experimentation. On the one hand, all related curriculums set curricular experimentation during lecture. On the other hand, we will choose some courses to let students take the consequent experiment in 17 and 18 semester. The former, the students learn the theoretical knowledge theory and basic software operations, which help the students comprehend the knowledge. The later deepened theory and enhanced software operation abilities.

\section{A. The Curricula Experimentation}

The curricular experimentations were allocated within the 16-week semester. All courses of big data and computer curriculums set curricular experimentation. Students learn the basic operations or the core operations in the curricular experimentation. The disadvantage of the curricula experimentation that there are fewer class hours. And the general class hours are 8-12 hours. The teachers only lectured the most basic knowledge and simple operations. Students mostly relied on their spare time to study. The teaching effect is not high.

\section{B. The Comprehensive Experimentation}

Between 16-week semesters, our institute arranged 3week semester, which is called the small semester. Some courses are launched 1-week or 2-week to finish project. Cooperative learning and Problem-based learning are mainly used this phase. In the first small semester, students is arranged the same course which emphasized operations of $\mathrm{C}$ and Internet-based software. In the second small semester, they is arranged python, database and Management 
Information System. In the third small semester, they is arranged data analysis and data mining. And students must finish a project in the third semester.

\section{CONCLUSIONS}

With computer and communication rapid development, Big data technology are applied every industry. On the other hand, students are demanded data analysis technology by the enterprise. So, it is important to find effectively cultivating big data and computer ability. This paper describes a curricular of information management and information system specialty at the Beijing Institute of Graphic Communication, Beijing, China. The courses divided into two threads in accordance with the basic skills and professional skills. The paper also introduces several effective pedagogical methods in order to increase practice ability, self-study ability and innovation ability of students. Experimentation is an important ability cultivating means, which train students a high level of software application ability. So this paper introduces experimentation arrangement. But we find some problems in curricular framework, which included courses arrangement, courses number, courses contents and etc. In the following, we still have to improve curriculum.

\section{ACKNOWLEDGMENTS}

This work was supported by the 2018 Course construction of Beijing Institute of Graphic Communication.

\section{REFERENCES}

[1] http://www.bigc.edu.cn/jgsz/jgsz1/.

[2] McAfee, Andrew; Brynjolfsson, Erik.Big Data: The Management Revolution. Harvard Business Review. Oct2012, Vol. 90 Issue 10, p60-68.

[3] Schrage, Michael.How the Big Data Explosion Has Changed Decision Making. Harvard Business Review Digital Articles. 8/25/2016, p2-5.

[4] Nigel Linge and David Parsons, "Problem-Based Learning as an Effective To Teaching Computer Network Design, "IEEE Transactions on Education,vol. 49, NO. 1,pp5-10, February 2006.

[5] Suzanne W. Dietrich and Susan D. Urban, "A Cooperative Learning Approach to Database Group Pjects: Integrating Theory and Practice,"IEEE Transaction on Education.vol.41,NO.4,November 1998.

[6] Eisenhardt. M., Kathleen, "Building theories from case study research 1989,"Academy of Management Review, vol.14, No.4, pp.532-550. 